\title{
THE ROLE OF SATELLITE EARTH OBSERVATION DATA IN MONITORING AND VERIFYING INTERNATIONAL ENVIRONMENTAL TREATIES
}

\author{
Shaida Johnston, NASA, Goddard Space Flight Center, Greenbelt, MD, U.S.A \\ Shaida.S.Johnston@nasa.gov
}

\begin{abstract}
The term verification implies compliance verification in the language of treaty negotiation and implementation, particularly in the fields of disarmament and arms control. The term monitoring on the other hand, in both environmental and arms control treaties, has a much broader interpretation which allows for use of supporting data sources that are not necessarily acceptable or adequate for direct verification. There are many ways that satellite Earth observation (EO) data can support international environmental agreements, from national forest inventories to use in geographic information system (GIS) tools. Though only a few references to satellite EO data and their use exist in the treaties themselves, an expanding list of applications can be considered in support of multilateral environmental agreements (MEAs). This paper explores the current uses of satellite Earth observation data which support monitoring activities of major environmental treaties and draws conclusions about future missions and their data use. The scope of the study includes all phases of environmental treaty fulfillment - development, monitoring, and enforcement - and includes a multinational perspective on the use of satellite Earth observation data for treaty support.
\end{abstract}

\section{INTRODUCTION}

Multilateral Environmental Agreements (MEAs) are typically brief and formal documents that describe the problem being addressed, the commitments of the governments involved, and the institutional infrastructure to be created. They are commonly cast in the form of binding international treaties, though some are non-binding statements of principles. Most MEAs create a series of international organizations to administer the agreement, such as secretariats and technical and scientific committees, and invest the power to alter and amend the treaty in a Conference of the Parties, which acts by consensus in almost all cases.

Though there is no universal definition of verification, the United Nations has devised a formula describing verification as a process that establishes whether the States parties are complying

- Copyright ${ }^{\circ} 2004$ by the International Astronautical Federation. All rights reserved. with their obligations under an agreement ${ }^{1}$. The process includes the collection of information relevant to obligations and agreements; analysis of the information; and reaching a judgment as to whether the specific terms of an agreement are being met. Nation states make available the information necessary for assessing their compliance. These declarations may be examined by other states or an international organization with respect to their reliability.

Verification, within the regimes of arms control and nuclear non-proliferation, is defined as an agreed process for determining contractual compliance by the contracting states. Verification may make use of predetermined technical instruments (measuring systems, surveillance instruments) and also other methods (inspections, reports and auditing). Compliance, in contrast, is established ex post facto and prevention of infringements is not the aim in that case, but rather the detection of infringements ${ }^{2}$. The goal of verification is to establish reciprocal certainty for the contracting states that contractual compliance is maintained.

The basic verification system of most environmental agreements involves nation states self-auditing their implementation of the agreement. Such a rudimentary approach differs in many respects from the verification of multilateral or bilateral arms control conventions. The lack of national or international inspectors exercising verification activities ${ }^{*}$ with their own measuring and surveillance instruments in the countries themselves is one central difference to modern arms control treaties. Fischer (1995) identifies several explanations for the weak verification provisions in environmental and resource protection conventions $^{3}$. One is that international environmental policy does not objectively affect any political field relevant to (inter) national security and power politics. Therefore there is little need for verification. Of course, the chances for international cooperation in general, and the

\footnotetext{
- Exceptions are the Antarctic Treaty (1959) and International Convention for the Prevention of Pollution from Ships (MARPOL), which both allow unrestricted on-site inspection as methods of verification.
} 
by measuring infrared light being emitted from an object, while a thermometer measures temperature directly within the source (in situ). Measurements of solar wind in space are performed in situ by having a spacecraft travel to the place of measurement and recording observations, while other satellites remotely sense the sun's surface. Most Earth parameters measured from space are done so 'remotely' by passive or active sensors in orbit around the Earth.

\section{MONITORING VS. VERIFICATION}

Verification of treaty compliance can be contrasted with monitoring, which is more varied and flexible in its goals. Verification is inherently a political process, since there must be political will to confront violations and is lacking in most MEA institutional structures ${ }^{6}$. Monitoring on the other hand serves multiple purposes, many of which need not focus on compliance or on the behavior of single states. Monitoring functions within the environmental treaty regime are also sometimes termed implementation reviews. Implementation, a concept related to both compliance and effectiveness, refers to the process of putting international commitments into practice. Implementation occurs at the international level through the establishment of organizations like secretariats and the holding of regularized meetings of the parties to an MEA. Implementation occurs
Domestically through the passage of legislation, promulgation of regulations, and enforcement of rules. This paper will address reviews in the context of three phases of treaty fulfillment development, monitoring and enforcement (Figure 1). The development phase includes initial awareness of an environmental problem and the defining of the problem that needs to be solved, along with the actual treaty negotiation process. Political will by nation states, and therefore public awareness, is required to develop an agreement to address global or regional scale environmental problems. These factors can be influenced by the existence and public sharing of scientific data, including Earth observation data. Reviews in the monitoring phase include reviews of national reports submitted from nation states, effectiveness reviews and environmental assessments ${ }^{\dagger}$. National implementation reviews are treated as the purview of nation states and are not addressed here. As treaties are evaluated for their effectiveness, and environmental assessments are regularly performed, there is opportunity for modifications or refinements to the MEAs. This feedback and refinement is part of an evolutionary development phase. Compliance reviews are part of the enforcement phase but are rarely performed for most MEAs.
DEVELOPMENT

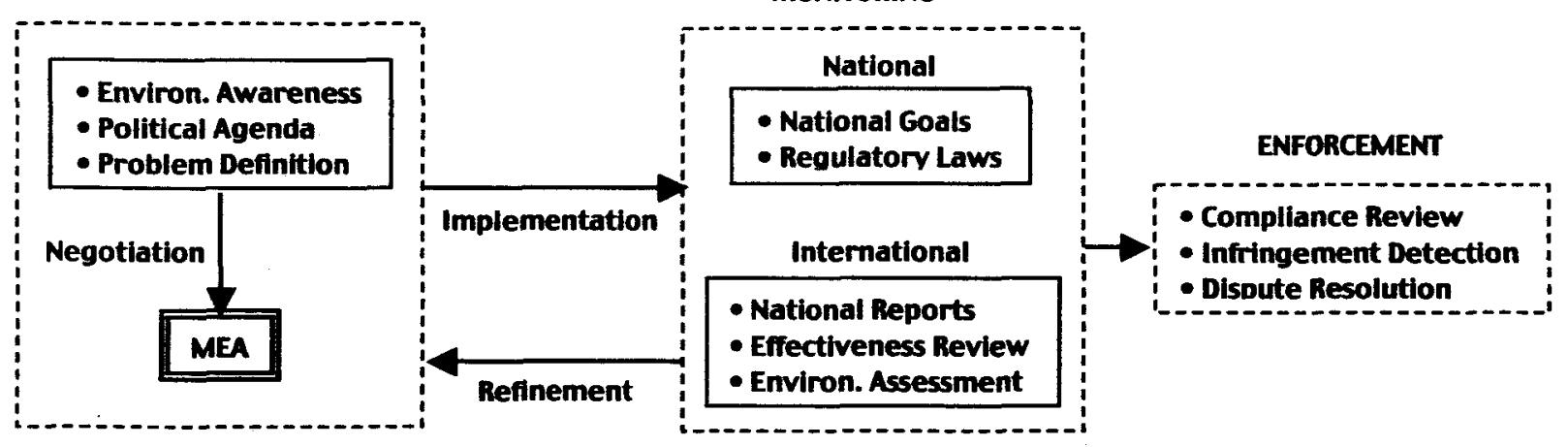

Figure 1 - Phases of MEA Fulfillment

\footnotetext{
:t The term "Systems for Implementation Review (SIR)" has also been use to address all aspects of MEA review, including compliance review and effectiveness review ${ }^{7,8}$.
} 
formation of international regimes in particular, are improved if verification instruments are available. Another difference between environmental treaties and those of weapons regimes is that the MEAs are trying to affect a behavior change in private sector actors - agricultural, energy, and transportation industries, and consumers. Through domestic policies affecting various forms of regulatory or economic practices, national goals and commitments are to be met. Arms control and weapons regimes need only deal with nation states and their public institutions to garner cooperation.

International conventions on environmental protection are receiving increasing interest from both policy makers and scientific disciplines concerned with transboundary problems. Today more than 350 MEAs exist that govern the cooperation of the participating nation states in protecting our common environment. Only a few have external monitoring functions explicitly written into the agreements, and fewer still incorporate or depend on Earth observation (EO) data to verify effectiveness or monitor compliance. MEAs mostly use nation-level self-monitoring techniques, such as registering inventories in a central repository or database, and internal state census of various biological species, as monitoring mechanisms, rather than external bodies or observations.

The interest in Earth observation or remote sensing as a tool for the development, monitoring and enforcement of environmental treaties, stems from parallel developments in the areas of Earth observation and international environmental diplomacy. Remote sensing technology and the rapid growth in the number of environmental treaties since the 1972 Stockholm Conference on the Environment have evolved on separate paths, converging only in the last ten years. On the one hand, instruments are being launched with ever more impressive capabilities, and vendors are looking for new markets. On the other, the numbers of treaties in force are constantly increasing, and decision makers, i.e., Contracting Parties are looking for easier ways to monitor their own and third party compliance 4 . For most of this growth period, scientists and decision-makers on both sides had little contact; remote sensing scientists knew little of the data needs of the treaty community, and treaty staff and contracting parties were often unaware of what remote sensing technologies were available or how they could provide information to the implementing organizations of the MEAs.
Several workshops in recent years ${ }^{\dagger}$ have been expressly organized to foster dialog, gradually bridging this gap. Challenges still exist in the technical, institutional, and political domains but signs exist that the critical integration of remote sensing technology and international environment policy is beginning to take place ${ }^{5}$.

The MEAs considered in this study are nine of the farthest reaching global treaties written to date: Convention on Wetlands of International Importance especially as Waterfowl Habitat (1971) [Ramsar Treaty]; Convention to Combat Desertification (1994) [CCD]; International Convention for the Prevention of Pollution from Ships (1973) [MARPOL]; Vienna Convention on the Protection of the Ozone Layer (1985) and its subsequent Montreal Protocol on Substances that Deplete the Ozone Layer (1997) [Montreal Protocol]; UN Framework Convention on Climate Change (1992) [UNFCCC] and its subsequent Kyoto Protocol (1997) [Kyoto Protocol]; Convention on Biological Diversity (1992) [CBD]; Convention on the Conservation of Migratory Species of Wild Animals (1979) [CMS]; Convention on International Trade in Endangered species of Wild Flora and Fauna (1973) [CITES]; and Antarctic Treaty (1959). This proliferation of MEAs has produced a new demand for environmental data and for better understanding of the socioeconomic processes and government policies that affect the environment. Remotely sensed data are critical to understanding Earth systems and human impacts on those systems, and can ultimately contribute to the design of improved policy instruments.

'Remote sensing' has become the short hand term for remote sensing of the Earth from space, but remote sensing applies to any measurement conducted remotely as distinguished from a measurement taken in situ. A thermal imager remotely senses temperature

\footnotetext{
${ }^{\dagger}$ March 2001 American Institute of Aeronautics and Astronautics (AIAA) workshop on International Space Cooperation in Seville, Spain, included a working group on the contribution of space systems to the development and implementation of MEAs; December 2000 workshop in Washington, D.C. entitled "Remote Sensing and Environmental Treaties: Building More Effective Linkages"; March 2000 African Association of Remote Sensing of the Environment in Cape Town, South Africa addressed using remote sensing data to support MEAs; and July 1999 United Nations University workshop in Tokyo, Japan entitled "InterLinkages: Synergies and Coordination between Multilateral Environmental Agreements".
} 


\section{DEVELOPMENT}

In 1962, the Kennedy administration released photos of Soviet missiles in Cuba taken from high-altitude airplanes. Those images shaped the course of the historic Cold War confrontations and demonstrated that remote sensing technologies could serve as powerful diplomatic tools.

Concern that chlorofluorocarbons (CFCs) might deplete the ozone layer, causing skin cancer and other health and ecological effects, dates back to 1974. Yet understanding of the problem changed significantly with the detection of the Antarctic ozone 'hole' in 1985 using the spaceborne Total Ozone Mapping Spectrometer (TOMS) ${ }^{* *}$. In the 1970s the United States, Canada, Norway, and Sweden acted unilaterally to control some uses of CFCs. International efforts included monitoring, research, and assessment programs beginning in the middle 1970s. The Vienna Convention (1985) established a framework for subsequent protocols; the Montreal Protocol (1987), negotiated and signed shortly after the ozone hole was detected, committed signatories to cut the planned use of offending chemicals by half. Since then, amendments and adjustments have been made to the protocol calling for a ban of ozone-depleting substances?.

Just as the proliferation of MEAs has increased the need for environmental data, so too remote sensing has created demand for better environmental law. Remote sensing is an unparalleled source of information that conveys environmental changes in a visually compelling way. As a result, it is extremely useful for raising awareness and developing the political support necessary to strengthen MEAs and environmental laws at the international level.

Beyond strictly technical applications remote sensing imagery can influence high-level political decisions that are directly relevant to treaty implementation. In1990, a four-million acre biosphere reserve straddling the border between Mexico and Guatemala was established in part due to evidence of tropical forest destruction gained through satellite data ${ }^{10}$. The Mexican side was largely deforested while the Guatemalan side held largely intact forest cover. The stark contrast at the border, clearly visible in a Landsat image, catalyzed the first meetings in decades between the Mexican and Guatemalan presidents to discuss

** Data from the TOMS instrument have been used extensively to tract Arctic and Antarctic ozone changes. The TOMS instrument flew on the Nimbus-7 (11/784/93), Meteor-3 (8/91-12/94), and Earth Probe (7/96$12 / 00$ ) satellites. border land management. The decision to set up the Mesoamerican Biological Corridor also stemmed from impact of imagery of the region. Remote sensing data demonstrated the ability to monitor large-scale change in the regional environment and helped create a climate in which regional environmental planning was possible. Remote sensing data had helped to raise the profile of deforestation in the first place by alerting the public and decision makers to the scope of the problem.

Environmental assessments may influence the understandings that actors have of an environmental problem by providing new data and analyses. They may also increase concern about a given issue, influencing its placement on the international agenda. Environmental assessments can influence implementation efforts, the terms of debate, and even their resolution, by framing an issue or by linking previously discrete problems. Assessments may also alter the political strategies of actors on specific problems and prompt the development of new policies and institutions. MEAs are evolutionary documents, intended to initiate a long-lasting cooperative process. This is part of the impetus behind the increasingly common framework-protocol format: the framework provides the ground rules and procedures for the creation of new, more detailed agreements. Environmental assessments play an important role in adjustment processes and may feed into review institutions. MEAs are evolving, open processes, which are continually reviewing implementation and developing new measures to improve effectiveness. The number of MEAs, particularly at the regional level will continue to grow and EO can greatly contribute to the ongoing development and refinement of MEAs by assisting in problem definition and catalyzing action.

\section{MONITORING}

Monitoring techniques range from relatively passive national reporting, as for most environmental agreements, to active on-site inspections for weapons and arms control treaties. Technology is used for making both in situ and remote measurements, from aerial or space borne platforms (e.g. national technical means $\left.{ }^{8}\right)$. Sensors can include anything from

\footnotetext{
${ }^{\S}$ National technical means (NTM) refers to satellite reconnaissance capabilities, which are simply another form of Earth observation.
} 
video equipment to seismographs, or visible, infrared (IR) and ultraviolet (UV) radiometers to active microwave radars.

Monitoring functions are performed by the secretariats of some MEAs. The more recent MEAs (Montreal and Kyoto Protocols) have more developed systems for monitoring, providing for the review of national reports and allowing environmental assessments to contribute to the evaluation of effectiveness. But even the Montreal Protocol is dependent on national reports of production, imports, and exports of ozonedepleting substances ${ }^{11}$. MEAs previously written with little monitoring or review mechanisms also have opportunities to strengthen their processes. Insofar as scientific information is critical for monitoring, the existing national scientific research programs - which are frequently not organized or funded for the explicit purpose of providing information to the regime - are the most important sources of information. Many MEAs are not currently gathering, requesting or using scientific data to any noticeable extent and some researchers studying MEAs and their implementation have expressed doubt that RS data will be treated any differently"*. Reporting and review provisions are very weak in most MEAs, and attempts to improve them, and to make them more rigorous and datarich, have largely failed in the past. The Montreal Protocol is a major exception to this general rule insofar as scientific data are being used extensively. But the political commitment is quite high for ozone protection, which suggests that political will is what is required for rigorous monitoring and review. The Montreal protocol has the most fully developed system for monitoring and review of all the MEAs. Implementation is regularly reviewed by a dedicated implementation committee, which also administers the noncompliance procedure of the protocol ${ }^{12}$.

\section{National Reporting}

Secretariats collect and make available national reports submitted by an MEA's contracting parties, but very little is done with most reports except collection. CITES compiles and uses the data from national reports to analyze patterns of behavior in trade in listed species, and for the CBD the reports are synthesized to identify general problems. In the biodiversity conventions CITES, CBD, CMS, and Ramsar - the national reports are not individually analyzed, while in

\footnotetext{
** Comment of O. Young at CIESIN workshop, December 2000
}

UNFCCC, reports from the major industrialized nations are systematically analyzed in detail. Some case studies and localized uses of EO data have begun to appear in individual national reports. Landsat data were used to prepare a land use map of tropical forests and wetlands of Sango Bay, Uganda, which was then combined with field survey data on plants and animals to prepare a biodiversity map. This biodiversity map was then used for conservation and planning, allowing more efficient national implementation of CBD and Ramsar for Uganda. The Ramsar convention will begin to see local case studies and pilot applications using EO data included in the national reports of Argentina, Bolivia, Brazil, Paraguay, Uruguay, Kenya, Senegal, South Africa, Canada, and the United States", though the reporting format needs to be expanded to allow the EO data to be included.

For CCD, there is currently no formal review by the committee of the parties (COP) of the national reports received, though they are looking to establish a formal implementation review committee. There is also no compliance and effectiveness review measures in the CCD. But the national reports of developed nations in 2002 each included some form of remote sensing data to support their summaries and research findings - maps of affected regions, GIS layers, or change detection images. CCD considers remote sensing "one of the modern tools that can help in combating desertification." The complexity of the causes of desertification sometimes leads to difficulties in assessing the magnitude of its effects and in making a detailed estimate of those locations where the phenomena manifest themselves. The use of satellite imaging techniques allows targeted assessments and correlations with the causes that are at the root of the phenomena. Many methods for estimating the physical phenomena associated with desertification call for the integrated use of high-resolution satellite images combined with low-resolution and high-frequency images like those of AVHRR ${ }^{: t+}$, and with geo-referenced ground surveys using satellite navigation systems implemented within regional geographic information systems. Inter-annual changes in the

\footnotetext{
It See the Ramsar Data Gateway at the Socioeconomic Data And Applications Center (SEDAC) website at http://sedac.ciesin.org/ramsardg/casestudies/index.html :t:t Advanced Very High Resolution Radiometer (AVHRR) provides daily coverage of the Earth's surface at a resolution of $1 \mathrm{~km}$.
} 
extent of desert areas, as measured by the normalized-difference vegetation index (NDVI), are easily measured using such data. Even though desertification can effectively be monitored from satellites, nations most involved in the CCD African and island nations - don't have satellite systems, access to EO data or the tools required to use the data. Capacity building for the $C C D$ is essential, and efforts to make data available and teach how to use them are integral to its success.

The Kyoto Protocol, when implemented, will require substantial data on greenhouse gas (GHG) emissions and carbon sources and sinks. Satellite sensors currently measure carbon monoxide*, methane, nitrous oxide and aerosols, but the technology is not at the point where it can easily inventory GHG emissions for a given country. Carbon emissions are monitored using proxy measures from fossil fuel consumption and estimates of biomass burning, rather than directly from the atmosphere. Carbon sequestration is monitored using proxy data also, in the form of forest inventories, agricultural data and ocean models. Remote sensing does provide valuable information on agricultural and forest land. The Australian Greenhouse Office, in support of UNFCC and the Kyoto Protocol, created a National Carbon Accounting System (NCAS) using multitemporal Landsat ${ }^{\dagger}$ data for the entire continent of Australia, from the period 1972-2000, to assess change in forest cover and productivity of vegetation communities across the continent. The sequence of images was chosen to provide maximum temporal resolution immediately before and after 1990 in order to achieve the best possible accuracy of land use change emissions in the (Kyoto) base year of 1990 . This example is just one of many national forest inventory activities occurring in nation states participating in the Kyoto Protocol. Reporting for the Kyoto Protocol will depend on accurate assessments of the 1990 base year, such that unless an aerial or ground-based inventory was developed in 1990 for a nation, satellite data from the that time frame will be needed. The data sets options therefore are

\footnotetext{
* $\mathrm{CO}$ is a proxy measurement for a number of GHGs.

t The Landsat series of satellites have been gathering moderate resolution $(30 \mathrm{~m})$ EO data and archiving them regularly since the first one was launched in 1972. Landsat data are the only long term source of Earth observation data that were acquired at a spatial resolution where man's impact on the environment, such as the harvesting of forest lands, can be evaluated accurately.
}

Landsat, Satellite Pour l'Observation de la Terre (SPOT) and AVHRR. Effectiveness Review

MEAs are only important if they achieve or produce outcomes that would not have otherwise occurred; that is to say they must be effective. Unlike national reports, an effectiveness review is not strictly focused on the actions of State parties individually, but rather look to the impact of actions collectively. Effectiveness review is not generally a regularized process in MEAs. In the case of the Montreal Protocol, EO data from the TOMS, GOME and POAM ${ }^{\dagger}$ instruments, along with ground-based total ozone measurements, have come to constitute regular effectiveness reviews providing daily and seasonal global ozone densities for comparison.

The Meso-American Biological Corridor which is a planned combination of protected areas and managed landscapes, that forms a continuous wildlife migration route from Panama to the Mexican border, supports CMS and CBD. As the region's major environmental initiative, the Corridor combines conservation and sustainable use of the biodiversity within the framework of sustainable development. The Corridor was mapped to monitor land cover using EO data (GOES, JERS-1, Landsat and MODIS data were used ${ }^{5}$ ) and to facilitate cooperative scientific research, data exchange, and training between U.S. National Aeronautics and Space Administration (NASA), participating universities and Central American researchers.

The European Commission's (EC) Global Monitoring for Environment and Security (GMES) initiative is producing a number of experimental products that demonstrate remote sensing capabilities in support of the Kyoto Protocol and the CCD. GMES is an effort of the EC to coordinate and expand the use of remote sensing in three primary areas: environmental treaties, natural disasters (especially floods and forest fires), and a third area grouping together environmental stress, population pressures and

\footnotetext{
:t Total Ozone Mapping Spectrometer (TOMS) flew on Nimbus-7, Meteor-3 and Earth Probe. Global Ozone Monitoring Experiment (GOME) currently flies on the second European Remote Sensing (ERS-2) satellite. Polar Ozone and Aerosol Measurement (POAM) was launched on SPOT-3 and most recently on SPOT 4.

${ }^{\S}$ Geostationary Operational Environmental Satellites (GOES); Japanese Earth Resource Satellite; Moderate Resolution Imaging Spectroradiometer (MODIS)
} 
humanitarian aid. For environmental treaties, GMES looks at applications tied to specific treaty provisions, such as land-based carbon sinks and emissions under the Kyoto Protocol. GMES began in 1998 as a technically driven initiative by the European space agencies, but has evolved since then into a politically accepted concept. What GMES has succeeded in doing is uniting the needs of various users in the European Union (EU) member governments, thereby providing a useful forum for interaction between the EC, government agencies involved in environmental negotiations or disaster preparedness, and the European space agencies. As part of GMES, the European Space Agency (ESA) supports Treaty Enforcement Services Using Earth Observation (TESEO), which funds research supporting remote sensing applications specific to the Ramsar Convention on Wetlands, the Kyoto Protocol, the Convention to Combat Desertification and MARPOL. The ESA seeks to develop a prototype aimed at demonstrating the capabilities of existing and future EO technology to support end users, e.g. treaty secretariats and contracting parties, in the implementation of these treaties.

\section{Environmental Assessments}

The primary link between environmental assessments and review institutions is in the area of effectiveness review. Assessments are important to effectiveness review because they provide data on changes in underlying environmental problems. In some cases, the integration of assessments into review works well; the Montreal Protocol system, for example, has relied on assessment of ozone depletion and of concentrations of ozone depleting substances in the stratosphere. For most MEAs however, assessments either do not exist or are not well integrated into review institutions. Because assessments are systemic in nature, rather than focused on individual governments or actors, the use of EO in assessment may be politically more palatable than even some effectiveness reviews.

The Millennium Ecosystem Assessment (MA) is the latest in a series of global integrated assessments. The MA consists of a global scientific assessment as well as a number of smaller, more focused regional, national, and local assessments. The primary users of MA results include the international ecosystem-related conventions - the CBD, CCD, and Ramsar - and their contracting parties. A much wider audience of United Nations (UN), governmental and nongovernmental agencies will also have the potential to refine their policies and programs based on the assessment results. Global in nature, consistent across nation boundaries, Earth observation data are integral to this effort. In conjunction with NASA's Global Land Cover data, the MA seeks to develop global coverage of processed EO data for the year 2000. This effort will classify ecosystem type and land use globally, and for some locations will be able to use a continuous variable (e.g. percent tree cover, percent grass cover, etc.) to describe the classification rather than categorical variables (e.g. forest/non-forest), adding detail and accuracy to the assessment.

The Intergovernmental Panel on Climate Change (IPCC) was established by World Meteorological Organization (WMO) and United Nations Environment Program (UNEP) to assess scientific, technical and socio-economic information relevant for the understanding of climate change, its potential impacts and options for adaptation and mitigation in support of UNFCC. To date, the Panel has produced three comprehensive assessments of global climate change $(1990,1995$ and 2001), covering scientific and technical information for researchers and policy makers. A fourth comprehensive assessment will be completed in 2007. The data are gathered, modeled and reported by scientists from around the globe, drawing on EO data, and historical and current records of ground-based measurements. Climate models are used to project impacts of current trends in climate change. The IPCC does not carry out research nor does it monitor climate related data or other relevant parameters. It bases its assessment mainly on peer reviewed and published scientific/technical literature. The First IPCC Assessment Report was completed in 1990 and the report played an important role in establishing the Intergovernmental Negotiating Committee for a UN Framework Convention on Climate Change by the UN General Assembly. The UNFCCC was adopted in 1992 and entered into force in 1994. The second assessment report, Climate Change 1995, provided key input to the negotiations, which led to the adoption of the Kyoto Protocol to the UNFCCC in 1997.

\section{ENFORCEMENT}

Because compliance review is more politically sensitive than other reviews, governments are often hesitant to engage in compliance review in the early years of an MEA. In no case where compliance review exists or is being considered was the system itself fully present in the original treaty text, though the original text often authorized the future creation of such a system. Some studies have argued that 
it would be preferable to negotiate substantive commitments together with their mechanisms for implementation and compliance review ${ }^{13}$. But there are significant political obstacles to doing so and there may be an inverse relationship between the stringency of review mechanisms and of substantive commitments ${ }^{14}$. It has been suggested often that EO data can be used for compliance reviews in the environmental regime ${ }^{15}$. The arguments cite science applications capable of detecting biological and physical phenomena that can point to potential treaty infractions, along with examples in the arms control regime of use of national technical means. These arguments suggest that simply because the data can be used for compliance verification, they should be. The effectiveness of external compliance verification in the environmental regime has proven to be a complex issue; touching on topics such as national sovereignty, information asymmetry and technical inequalities of the parties and credibility and continuity of EO data.

Remote sensing cannot provide a single source solution to information needs for any of the reviews discussed here. Rather it serves to complement an on-going program of in situ observations and national self-assessments. The promise of remote sensing is to allow the development of more robust and efficient ground sampling strategies and to subsequently extrapolate from such in situ measurements in both space and time. As with arms control and weapons treaties, EO data provide the larger spatial context that allows the limited resources of monitoring and verification teams to be focused toward areas of potential concern. It is relatively rare that a single piece of evidence gathered by a single monitoring system can be the basis for a charge of violation. Much more often the individual bits of evidence are ambiguous when taken separately and only acquire significance when assembled together in a pattern with other ambiguous bits of evidence.

An example of an application tied to treaty enforcement is the use of synthetic aperture radar (SAR) for marine oil spill detection, currently taking place under the auspices of the Bonn Agreement among the nations bordering the North Sea within the context of the MARPOL treaty. Under the Bonn Agreement, monitoring procedures have been set up to track oil spills to the ships of origin. Because oil slicks change the surface roughness of water bodies under windy conditions that generally prevail on high seas, this registers as changes in backscatter on radar instruments. SAR images have proven useful for spill monitoring ${ }^{16}$. However, radar images generally give an unacceptable number of false positives, so the technology is only applicable as a surveillance tool in conjunction with aerial IR and UV sensors used for reconnaissance and confirmation of potential oil slicks. Under the Bonn Agreement, photographic evidence is till required in order to bring a ship's owner to prosecution. The advantage of space-based SAR is that it can cover a huge expanse of ocean targeting the limited surveillance resources of aircraft equipped with passive IR and UV sensors and photographic equipment toward areas suspected of having experienced an oil spill.

\section{CONCLUSION}

The dialog has begun. The remote sensing community is recognizing the MEA community as an important user of their data and products, and has started to understand the needs and importance of the international environmental treaty regime. Reciprocally, contracting parties of MEAs are recognizing the insights and value provided by EO data. Where some degree of caution is required while proceeding with this dialog, is in deciding where EO can most effectively be used. During the development and refinement of treaties, EO data have proven to be quite effective. Effectiveness reviews and environmental assessments have had positive impacts and EO data are being embraced as essential to those processes. Verification and compliance reviews, on the other hand, are met with reticence, whether using EO data or not. Sovereignty concerns take precedence over enforcement of treaty provisions, and contracting parties therefore are unwilling to accept external verification. Until global or regional threats from environmental change are perceived to significantly affect national interests, states are unlikely to accept strict enforcement of treaties by third parties. Compliance is one area where governments are very sensitive and apart from certain cases, such as the Kyoto Protocol, EO data will not be effective for verification or compliance reviews. The remote sensing community should acknowledge these limitations when proposing MEA uses of EO data and spend their efforts where EO data are most useful. In the area of systematic monitoring of MEA effectiveness or ecosystem assessments, EO data are acknowledged to provide a valuable contribution to the regime. This is where efforts to join the communities will be most fruitful. 


\section{REFERENCES}

1. Kline, K. and Raustiala, K., International Environmental Agreements and Remote Sensing Technologies, for Workshop on Remote Sensing and Environmental Treaties: Building More Effective Linkages, Washington, D.C., 2000

2. Fischer, W., The Verification of International Conventions on Protection of the Environment and Common Resources, Forschungszentrum Jülich, 1991

3. Fischer, W., Hoffmann, H.-J., Katscher, W., Kotte, U., Lauppe, W.-D., and Stein, G., Agreements on Climate Protection - The Verification Problem, Forschungszentrum Jülich, 1995

4. de Sherbinin, A., Kline, K., and Raustiala, K., Remote Sensing Data: Valuable Support for Environmental Treaties, Environment 44 (1), 20-31, 2002

5. de Sherbinin, A. and Giri, C., Remote Sensing in Support of Multilateral Environmental Agreements: What Have We Learned from Pilot Applications?, for Open Meeting of the Human Dimensions of Global Environmental Change Research Community, Rio de Janeiro, 2001

6. Salt, J. and Lanchbery, J., Remote Sensing as a Tool for Verifying Compliance with Environmental Agreements, in Verification 1994 Arms Control. Peacekeeping and the Environment, Poole, J. B. and Guthrie, R., Brassey, 1994
7. Victor, D., Raustiala, K., and Skolnikoff, E., The Implementation and Effectiveness of International Environmental Commitments: Theory and Practice, MIT Press, 1998

8. Raustiala, K., Reporting and Review Institutions in 10 Multilateral Environmental Agreements, UNEP, 2001

9. Ausubel, J. H. and Victor, D. G., Verification of International Environmental Agreements, Annual Review of Energy and Environment 17, 1-43, 1992

10. Kline and Raustiala

11. Ausubel and Victor

12. Raustiala

13. Victor, Raustiala and Skolnikoff

14. Szell, P., The Development of Multilateral Mechanisms for Monitoring Compliance, in Lang, W. (ed.), Sustainable Development and International Law, Graham and Trotman, 1995

15. CIESIN, Remote Sensing and Environmental Treaties: Building More Effective Linkages, Report of a Workshop, Washington, D.C., December 2000

16. Jones, B., A Comparison of Visual Observations of Surface Oil with Synthetic Aperture Radar Imagery of the Sea Empress Oil Spill, International Journal of Remote Sensing, 22 (9), 1619-1638, 2001 